\title{
Economic Analysis of the Use of Anti-DFS70 Antibody Test in Patients with Undifferentiated Systemic Autoimmune Disease Symptoms
}

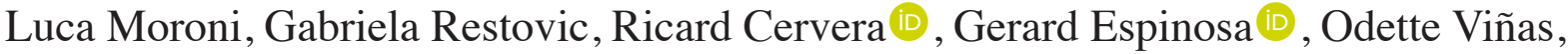 \\ Milagros García, and Laura Sampietro-Colom
}

\begin{abstract}
Objective. In patients with antinuclear antibodies (ANA) and undifferentiated features of systemic autoimmune disease, the coexistence of monospecific anti-dense fine speckled 70 (anti-DFS70) antibodies is associated with a lower risk of progression to overt disease. Therefore, they might help in correctly classifying ANA- positive patients and avoiding unnecessary followup diagnostic procedures. The aim of this study was to analyze the economic effect of the introduction of the anti-DFS70 antibody test in a hospital setting.

Methods. A case-control study was performed to detect monospecific anti-DFS70 antibodies in ANA-positive subjects with undifferentiated features (cases, $n=124$ ) and with a defined systemic autoimmune disease (controls, $\mathrm{n}=290$ ). Based on current clinical practice, a decision tree was developed to represent the disease course of patients with undifferentiated features in the subsequent 3 years. A budget impact analysis (BIA) was performed to estimate the effect of implementing the screening for anti-DFS70 antibodies in the case group on the total costs. A sensitivity analysis was conducted to calculate the effect of the uncertainty of the input variables on the results.

Results. Among the 124 patients in the case group, 5 (4.0\%) tested positive for anti-DFS70 antibodies versus $4 / 290(1.4 \%)$ in the control group ( $\mathrm{p}=$ not significant). The mean cost per patient under the current clinical practice decreased from $€ 3274$ to $€ 3192$ in our scenario. The BIA reports cost savings of $€ 10,128$.

Conclusion. The introduction of anti-DFS70 antibody test would avoid unnecessary followup diagnostic procedures and minimize the use of health resources generated by suspicion of a potential systemic autoimmune disease. (First Release April 15 2020; J Rheumatol 2020;47:1275-84; doi:10.3899/jrheum.190533)
\end{abstract}

Key Indexing Terms:

ANTI-DFS 70 ANTIBODY

UNDIFFERENTIATED CONNECTIVE TISSUE DISEASE SYSTEMIC AUTOIMMUNE DISEASE ANTINUCLEAR ANTIBODIES ECONOMIC EFFECT

\footnotetext{
From the Department of Autoimmune Diseases, the Department of Immunology, and the Assessment of Innovations and New Technologies Unit, Hospital Clínic, University of Barcelona, Barcelona; Fundació Clínic per la Recerca Biomèdica, Barcelona; Institut d'Investigacions Biomèdiques August Pi i Sunyer (IDIBAPS), Barcelona, Catalonia, Spain, the Unit of Immunology, Rheumatology, Allergy and Rare Diseases, San Raffaele Scientific Institute, Milan, Italy.

This study was funded by the Hospital Clinic Foundation and Werfen, Barcelona, Spain. The latter funders had no role in the study design, data collection, data analysis, data interpretation, or writing of the report. The authors had full access to all the data in the study, had final responsibility for the decision to submit for publication, and take responsibility for the integrity of the work as a whole.

L. Moroni, MD, Department of Autoimmune Diseases, Hospital Clínic, University of Barcelona, and Unit of Immunology, Rheumatology, Allergy and Rare Diseases, San Raffaele Scientific Institute; G. Restovic, MD, Fundació Clínic per la Recerca Biomèdica; R. Cervera, $M D, P h D$, FRCP, Department of Autoimmune Diseases, Hospital Clínic, University of Barcelona, and IDIBAPS; G. Espinosa, MD, PhD, Department of Autoimmune Diseases, Hospital Clínic, University of Barcelona, and IDIBAPS; O. Viñas, MD, Fundació Clínic per la Recerca Biomèdica, and Department of Immunology, Hospital Clínic, University of Barcelona; M. García, MD, Fundació Clínic per la Recerca Biomèdica, and Department of Immunology, Hospital Clínic, University of Barcelona; L. Sampietro-Colom, MD, Assessment of Innovations and New
}

Technologies Unit, Hospital Clínic, University of Barcelona.

Address correspondence to Dr. R. Cervera, Department of Autoimmune Diseases, Hospital Clínic, Villarroel, 170, 08036-Barcelona, Catalonia Spain.E-mail: rcervera@clinic.cat

Accepted for publication October 11, 2019.

Antinuclear antibodies (ANA) are the hallmark of most systemic autoimmune diseases. Although ANA testing is widely used, its low specificity represents a significant pitfall $^{1}$. Recently, a new ANA specificity, called anti-dense fine speckled (DFS) 70 antibody, has been identified in patients with no or few symptoms of systemic autoimmune disease but with positive ANA by indirect immunofluorescence (IIF) ${ }^{2}$. The anti-DFS70 antibodies recognize the 75-kDa lens epithelium-derived growth factor, an autoantigen associated with a DFS IIF staining pattern on HEp-2 cells $s^{1,3,4,5}$.

Anti-DFS70 antibodies are responsible for as much as $12 \%$ of positive HEp- 2 results in a routine setting ${ }^{6}$, and they have been associated with a wide variety of conditions such

Personal non-commercial use only. The Journal of Rheumatology Copyright $\subset$ 2020. All rights reserved. 
as Vogt-Koyanagi-Harada syndrome, atopic dermatitis, and asthma ${ }^{2}$. Currently, they are considered to be more prevalent in patients without systemic autoimmune diseases and healthy individuals ${ }^{7,8}$, and although their presence cannot exclude the existence of systemic autoimmune disease, the probability is significantly lower ${ }^{7}$. Patients with systemic autoimmune diseases may have anti-DFS70 antibody associated with other immunological markers of autoimmune disease, although the isolated positivity of anti-DFS70 antibody is rare in these patients ${ }^{7,9}$. Therefore, the presence of isolated anti-DFS70 antibody could be useful to rule out a diagnosis of definite systemic autoimmune disease ${ }^{10}$. In addition, the introduction of the anti-DFS70 antibody test as an exclusion marker for systemic autoimmune diseases in diagnostic algorithms could be cost-effective ${ }^{11}$. However, in most studies showing epidemiological aspects of the antibody, the information on its economic effect is lacking $6,7,12,13$.

The main purpose of our present study was to determine the prevalence of anti-DFS70 antibodies in a cohort of patients with undifferentiated features of systemic autoimmune diseases with ANA positivity but negative antigenic specificity, and the economic effect of the implementation of anti-DFS70 antibody test in the management of these patients compared to the current clinical practice.

\section{MATERIALS AND METHODS}

Clinical study design. A clinical retrospective case-control study was carried out at the Hospital Clinic of Barcelona. The clinical study was designed to assess the prevalence and clinical associations of anti-DFS70 antibodies in ANA-positive patients without systemic autoimmune diseases (cases) and with definite systemic autoimmune diseases (controls). Patients in both groups were chosen consecutively among those attending the outpatient clinic at the Department of Autoimmune Diseases of the Hospital Clinic of Barcelona from January 1 to December 31, 2016.

Patients. Cases were defined as patients with positive ANA (HEp-2) at significant titer $(\geq 1: 80$ ), negative for antigenic specificities including anti-dsDNA, anti-Ro/SSA, anti-La/SSB, anti-Sm, anti-RNP, anti-Scl70, anticentromere, anti-RNA-polymerase III, and anti-PM/SCl antibody, and with a clinical suspicion of systemic autoimmune disease but without a definite diagnosis over 6 months from the beginning of the symptoms. This case definition greatly overlaps the most important proposed classification criteria for undifferentiated connective tissue disease (UCTD) ${ }^{14,15,16,17}$.

Four groups of patients were considered as control groups and included those fulfilling the currently proposed classification criteria of systemic lupus erythematosus (SLE) $)^{18}$, Sjögren syndrome (SS) ${ }^{19}$, systemic sclerosis $(\mathrm{SSc})^{20}$, and antiphospholipid syndrome (APS) ${ }^{21}$.

The study was approved by the local institutional review board (approval number: $\mathrm{HCB} / 2016 / 0790$ ) that waived the requirement for individual informed consent.

Variables. Clinical variables for the cases included symptoms or signs suggestive of systemic autoimmune diseases such as Raynaud phenomenon (RP), arthralgia, arthritis, fatigue, skin lesions, oral and genital ulcers, photosensitivity, fever, sicca syndrome, serositis, leukopenia, thrombocytopenia, livedo reticularis, thrombosis, and obstetric morbidity including early miscarriage ( $\leq 10$ weeks of pregnancy) and late abortions or fetal loss (> 10 weeks of pregnancy). Considering controls, the main clinical manifestations of the previously defined systemic autoimmune diseases were also collected. For cases and controls, comorbidities, associated organ-specific autoimmune diseases, and malignancies were registered. Followup was defined as the time (in months) from the beginning of the symptoms to the last visit. Immunological profiles, number of visits, and diagnostic imaging techniques in cases and controls, following the current routine care protocols established for each disease, were recorded to describe the health resources used by each group.

Chemiluminescence anti-DFS70 antibody assays. In all cases and controls, anti-DFS70 antibody determination by chemiluminescence (QUANTA Flash, Inova Diagnostics) was performed. The QUANTA Flash DFS70 assay is a novel chemiluminescence immunoassay that uses recombinant DFS70 expressed in Escherichia coli coated onto paramagnetic beads and is designed for the BIO-FLASH instrument (Biokit S.A.)7. The relative light units (RLU) are proportional to the amount of isoluminol conjugate that is bound to the anti-human IgG, which in turn is proportional to the amount of anti-DFS70 antibodies bound to the antigen on the beads. Using a standard curve, all RLU values are converted into calculated units (CU). Samples with antibody titers above the analytical measuring range (3.2-450.8 calculated units; cutoff $=20 \mathrm{CU}$ ) were prediluted 1:20 and retested to determine the exact anti-DFS70 antibody concentration.

Statistical analysis. Descriptive statistics were used to summarize all demographic and laboratory variables of both groups. Comparison of frequencies among groups for categorical variables was performed by chi-square and Fisher's exact tests. Nonparametric Kruskal-Wallis test was used to compare multiple median values of continuous non-normal variables among groups. Spearman rank model was used for correlation analysis. Receiver-operating characteristic curves were built to analyze diagnostic performance of biomarkers and scores. $\mathrm{P}$ values $<0.05$ were considered significant.

Economic analysis. Based on the results and the information collected in the clinical retrospective case-control study (i.e., the prevalence of anti-DFS70 antibodies in ANA-positive patients without systemic autoimmune diseases, health resources used per patients, and its unit costs), 2 types of economic analyses were carried out to estimate the effect of introducing the anti-DFS70 antibody test as a diagnostic tool in clinical practice.

The first analysis was a mean cost-per-patient analysis. A decision tree was developed ${ }^{22}$ to represent the disease course in the next 3 years of patients with symptoms of undifferentiated systemic autoimmune diseases from the moment they have been referred to the hospital to be diagnosed. The branches of the initial decision node represent the strategies to be compared. In this analysis, we are comparing the current clinical management at the hospital (current clinical practice without anti-DFS70) with the expected clinical management at the hospital after including the anti-DFS70 antibody test (expected scenario including anti-DFS70; Figure 1).

Literature data show clearly that patients with an undifferentiated onset will have 3 potential clinical courses: evolving to resolution of symptoms after 3 years' followup, remaining with unspecific symptoms after 3 years' followup, or developing a defined systemic autoimmune disease after 3 years' followup ${ }^{23}$. When the anti-DFS70 antibody test is included in the pathway of care to identify the presence of the systemic autoimmune disease in all patients with signs and symptoms of these diseases, a new potential health state is included in the disease course. This new health state - "anti-DFS70 positive with low possibility to develop a systemic autoimmune disease" - appears when patients test positive for anti-DFS70 antibody.

For all the health states (excluding the above-mentioned new health state), economic variables, considering the type and frequency of resources used for patients, were obtained from hospital records within the case-control study and validated by clinical experts (RC, GE). Economic variables included were laboratory tests, visits (first and followup), diagnostic imaging, and costs related to the referrals to other physicians. In this way, mean costs per health state were calculated. For the new health state, "positive anti-DFS70 antibody linked to low possibility to develop a systemic autoimmune disease," the types of resources to be used by these patients were obtained from the clinical experts' opinions, and they include the following: a first visit to a specialist, 1 followup visit, and 1 test including blood cell count, hepatic, and renal function tests, urinalysis, ANA HEp-2,

Personal non-commercial use only. The Journal of Rheumatology Copyright @ 2020 . All rights reserved. 


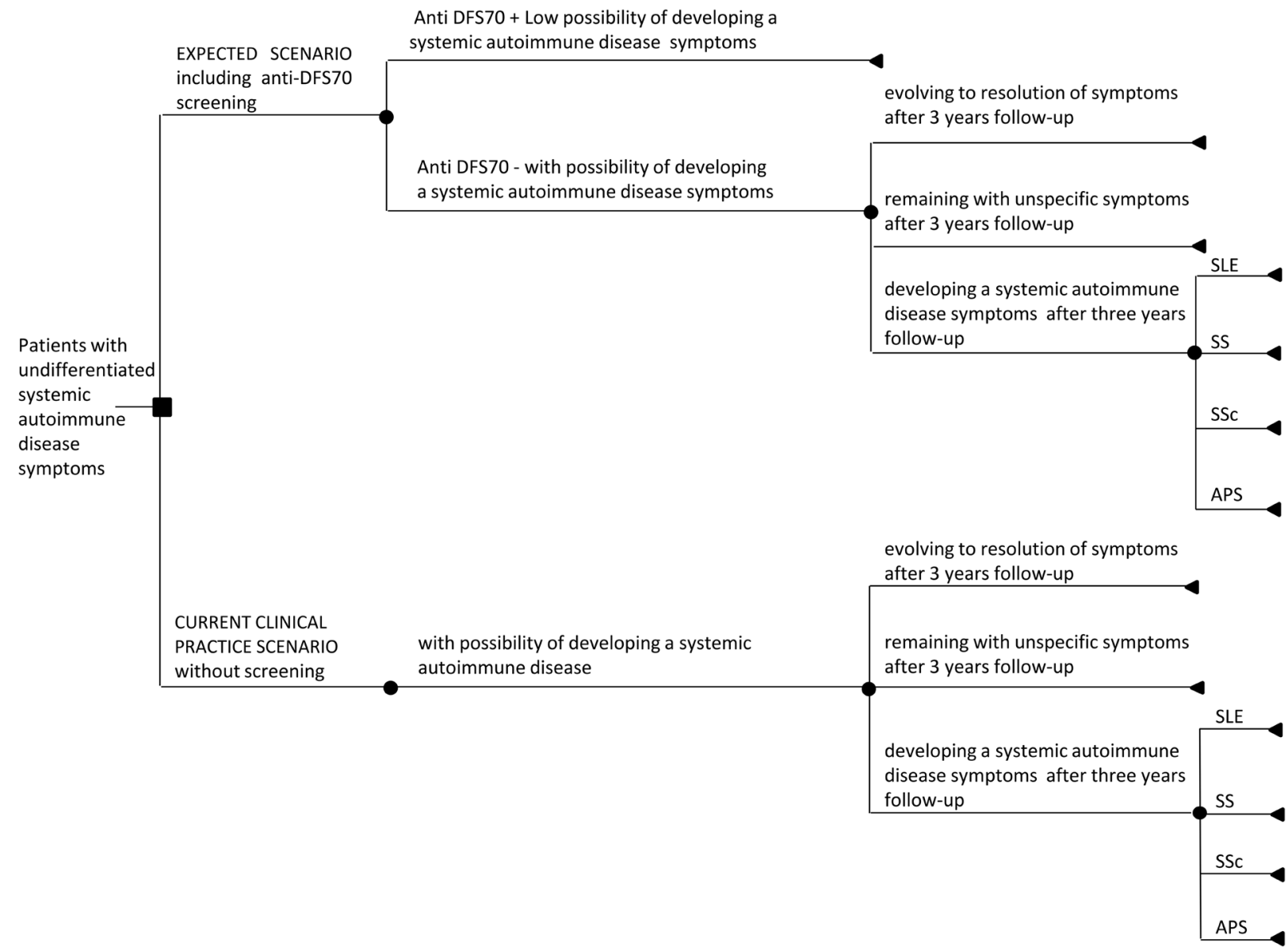

Figure 1. Decision tree showing the disease course (i.e., health states) in patients with undifferentiated systemic autoimmune disease symptoms under current clinical practice and when the anti-DFS70 test is used. Anti-DFS70: anti-dense fine speckled 70; SLE: systemic lupus erythematosus; SS: Sjögren syndrome; SSc: systemic sclerosis; APS: antiphospholipid syndrome.

anti-dsDNA/Crithidia luciliae, anticentromere, anti-Sc170, anti-Ro/SSA, anti-LA/SSB, and anti-Sm/RNP antibodies; and C3, C4, and CH50 levels. Moreover, clinical experts agree that under this new health state, the patient will be discharged and not followed systematically during the next 3 years. The transition probabilities for each health state (probability of moving from one state to another) to populate the decision tree were mainly obtained from the results of the clinical case-control study. When no data were available, information from the literature along with clinical expert judgment were used.

The second analysis was a cohort budget impact analysis (BIA), which is based on estimating the difference in the total costs per a cohort of patients using current clinical practice sequence or after the introduction of the anti-DFS70 antibody test as a diagnostic tool (expected practice). The clinical variable used here was the prevalence of anti-DFS70 antibody found in the case group of the clinical study. The total costs for current clinical practice were computed by first considering the 3 health states described above; then, multiplying by the average per-patient costs for each health state; and finally, summing across the results from each branch of the decision tree model. The total costs for the expected practice (introducing anti-DFS70 antibody test) were estimated in the same way adding the cost per patient of the already-described new health state ("anti-DFS70 positive linked to low possibility to develop a systemic autoimmune disease"). The difference between both practices allowed the estimation of the effect on the hospital budget.
Both analyses were performed from the perspective of the Hospital Clinic of Barcelona (considering only direct costs) and developed using Microsoft Excel. Costs not directly related to the diagnosis of the systemic autoimmune disease were not considered. Unit costs associated to the resources used were obtained from a hospital database and are expressed in 2018 Euros $(€)$. No discount rate was applied. The price of anti-DFS70 antibody test for the Hospital Clinic Barcelona was used.

One-way deterministic sensitivity analysis was conducted to analyze the effect of the uncertainty of economic and clinical variables on the final results. Sensitivity analysis is an important part of the evaluation process and gives valuable information to decision makers about the robustness of the findings of an economic evaluation, as well as the potential value of collecting more information before making a final decision ${ }^{24}$.

The main input variables used in the BIA ( $\%$ of patients with positive anti-DFS70 antibody, \% of patients evolving to resolution of symptoms after 3 yrs' followup, $\%$ of patients remaining with unspecific symptoms after 3 yrs' followup, $\%$ of patients developing a systemic autoimmune disease after 3 yrs' followup, and unit cost of the anti-DFS70 antibody test) were varied in a range of uncertainty. This was designed as a tornado graphic representation, which highlights the variables that may mostly affect the final results if the figures used in our analysis are different in other settings. The range of uncertainty (variation interval) for each variable was determined based on a range of variability equal to $\pm 20 \%$ of the central value, which represents a reasonably wide range. 


\section{RESULTS}

Clinical results from case-control study. Overall, 414 patients (124 cases and 290 controls) were included in the study. Four groups of patients were considered as control groups, including 91 patients with SLE, 82 with SS, 58 with SSc, and 59 with APS. As expected, case and control groups are predominantly female. The main clinical features of the patients in case and control groups are summarized in Table 1A. The most common "undifferentiated" symptom was arthralgia (40.3\%), followed by RP (32.2\%), and sicca syndrome (31.5\%). Distribution of ANA titers, ANA patterns, and extractable nuclear antigen (ENA) specificities by group are summarized in Table 1B.

Table 1A. Clinical manifestations of the studied population.

\begin{tabular}{|c|c|}
\hline Variables & $\mathrm{N}(\%)$ \\
\hline \multicolumn{2}{|l|}{ Cases, $\mathrm{n}=124$} \\
\hline Arthralgia & $50(40.3)$ \\
\hline $\mathrm{RP}$ & $40(32.2)$ \\
\hline Sicca syndrome & $39(31.5)$ \\
\hline Fatigue & $28(22.6)$ \\
\hline Leukopenia & $26(21.0)$ \\
\hline Obstetric morbidity & $26(21.0)$ \\
\hline Arthritis & $19(15.3)$ \\
\hline Skin lesions & $18(14.5)$ \\
\hline Aphthous ulcers & $17(13.7)$ \\
\hline Photosensitivity & $14(11.3)$ \\
\hline Fever & $14(11.3)$ \\
\hline Thrombocytopenia & $9(7.3)$ \\
\hline Serositis & $6(4.8)$ \\
\hline Thrombosis & $6(4.8)$ \\
\hline Livedo reticularis & $2(1.6)$ \\
\hline \multicolumn{2}{|l|}{ SLE, $n=91$} \\
\hline Articular involvement & $75(82.4)$ \\
\hline Cutaneous involvement & $68(74.7)$ \\
\hline Hematological involvement & $46(50.5)$ \\
\hline Renal involvement & $44(48.4)$ \\
\hline Serositis & $22(24.2)$ \\
\hline $\mathrm{RP}$ & $11(12.1)$ \\
\hline Fever & $9(9.9)$ \\
\hline Neuropsychiatric lupus & $6(6.6)$ \\
\hline \multicolumn{2}{|l|}{ Sjögren syndrome, $\mathrm{n}=82$} \\
\hline Glandular involvement & $64(78.0)$ \\
\hline Extraglandular involvement & $18(22.0)$ \\
\hline \multicolumn{2}{|l|}{$\mathrm{SSc}, \mathrm{n}=58$} \\
\hline Limited SSc & $34(58.6)$ \\
\hline Sine scleroderma & $14(24.1)$ \\
\hline Diffuse SSc & $8(13.8)$ \\
\hline Pre-scleroderma & $2(3.4)$ \\
\hline $\mathrm{RP}$ & $56(96.6)$ \\
\hline Gastrointestinal involvement & $41(70.7)$ \\
\hline Interstitial lung disease & $19(32.8)$ \\
\hline Articular involvement & $15(25.9)$ \\
\hline Pulmonary hypertension & $10(17.2)$ \\
\hline \multicolumn{2}{|l|}{ APS, $n=59$} \\
\hline Thrombotic & $45(76.3)$ \\
\hline Obstetric & $26(44.1)$ \\
\hline
\end{tabular}

RP: Raynaud phenomenon; SLE: systemic lupus erythematosus; SSc: systemic sclerosis; APS: antiphospholipid syndrome.
Prevalence of anti-DFS70 antibody. Considering the manufacturer cutoff value, 5 patients (4.0\%) among the 124 in the case group tested positive for anti-DFS70 antibody versus 4/290 (1.4\%) in the control group (1 for each disease, respectively; $\mathrm{p}=$ not significant). All 5 anti-DFS70 positive subjects in the case group showed homogeneous or homogeneous-speckled patterns (Figure 2). None of the 4 patients who tested positive for anti-DFS70 antibody in the control group were "monospecific" and displayed either at least 2 ENA specificities other than anti-DFS70 or anti-thyroperoxidase antibody (data not shown). No statistically significant difference in clinical profile was found in cases grouped by anti-DFS70 antibody status, nor in anti-DFS70 titer between positive cases and controls (163.7 CU vs $99.8 \mathrm{CU}$, respectively). Individual values of Quanta Flash DFS70 assay are reported in Table 1C, showing that none of the anti-DFS70 positive cases were displaying highest titers (1:640 or above).

Mean cost per patient analysis. The transition probabilities for each health state depending on the results of anti-DFS70 antibody (positive or negative) are shown in Table 2. The mean costs per patient under the current clinical practice and under the expected scenario including anti-DFS70 antibody as a diagnostic tool are shown in Table 3 . In the base case, the mean costs per diagnosed patient were $€ 3274$ under current clinical practice and $€ 3192$ when anti-DFS70 antibody test was incorporated as a diagnostic tool. Savings associated with the anti-DFS70 antibody test were fully explained by the fewer health resources used by patients who tested positive; these patients are discharged after the testing, saving health resources by not requiring additional laboratory tests, diagnostic imaging tests, and/or visits in the following 3 years.

Cohort BIA. The results of the 3-year followup of patients in each scenario are shown in Table 4. For the studied population, i.e., the 124 patients with signs and symptoms of undifferentiated systemic autoimmune disease, the total cost would be $€ 135,323$ per year for the next 3 years under the current clinical practice. The main cost corresponds to the laboratory test used in the diagnosis and monitoring of patients with positive ANA (HEp-2) at significant titer (1:80) without specific antibodies and without associated systemic autoimmune disease. For the expected sequence of events, the total costs would be $€ 136,108$ in the first year and $€ 129,866$ in the following 2 years. Therefore, the introduction of the anti-DFS70 antibodies as a diagnostic tool will have a cost reduction of $€ 10,128$ during the 3 years' management for the total number of patients analyzed in the study.

The deterministic 1-way sensitivity analysis (Figure 3) showed that the results were robust with no single variable modifying the finding that the introduction of the anti-DFS70 antibody test as a diagnostic tool for patients with undifferentiated systemic autoimmune disease was cost-saving

Personal non-commercial use only. The Journal of Rheumatology Copyright $\odot$ 2020. All rights reserved. 
Table $1 B$. Autoantibody distribution in the study population by disease group.

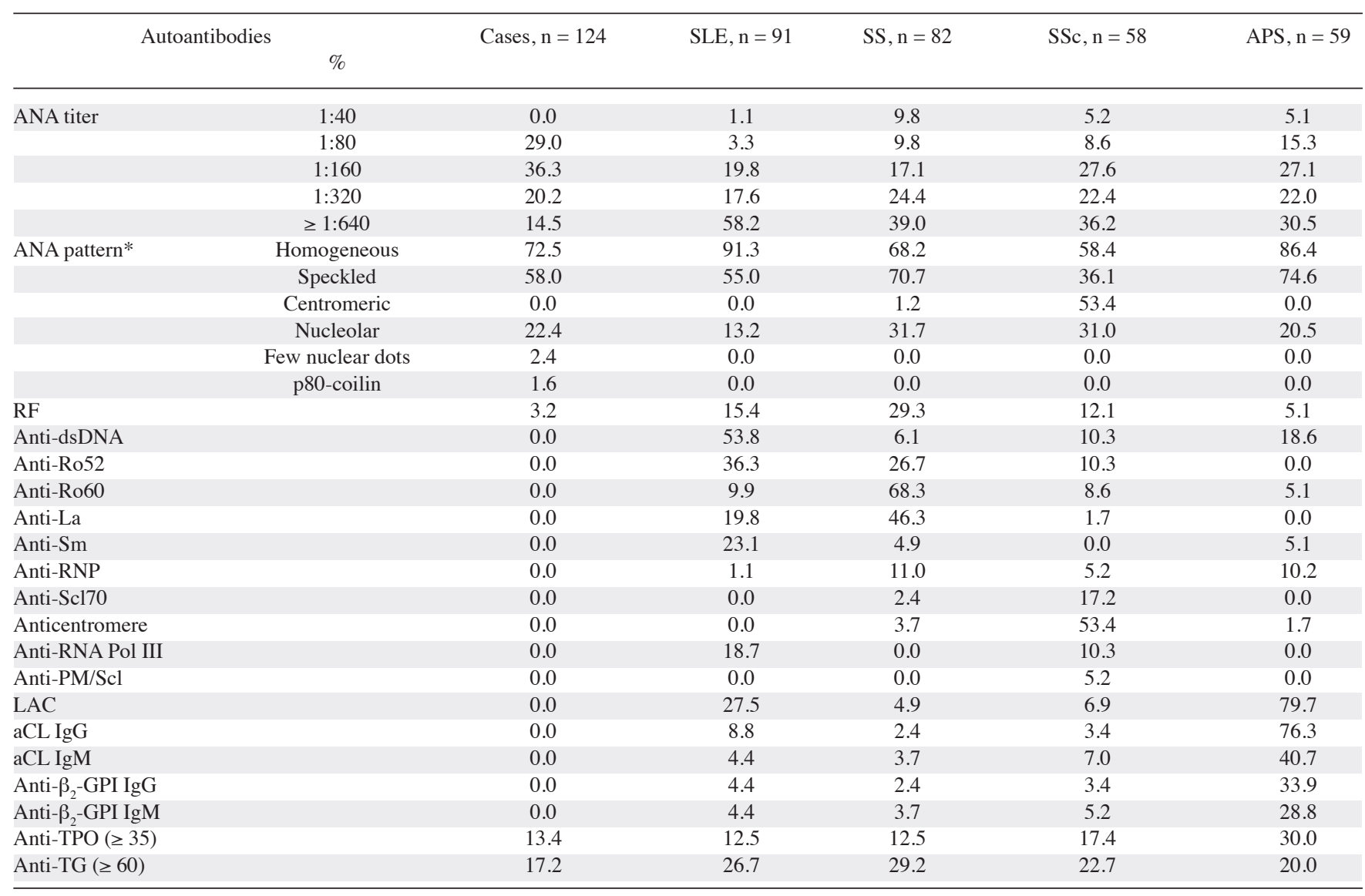

* Mixed patterns are included and counted twice. SLE: systemic lupus erythematosus; SS: Sjögren syndrome; SSc: systemic sclerosis; APS: antiphospholipid syndrome; ANA: antinuclear antibody; RF: rheumatoid factor; LAC: lupus anticoagulant; aCL: anticardiolipin antibodies; anti- $\beta_{2}$-GPI: $\beta_{2}$-glycoprotein I; IgG: immunoglobulin G; anti-TPO: antithyroid peroxidase antibody; anti-TG: antithyroglobulin.

compared with the current clinical practice. It is important to note that the prevalence of patients with anti-DFS70 antibody is the variable that most affects the differences of total costs found between the 2 scenarios: the higher the prevalence, the greater the cost reduction in the management of patients with undifferentiated systemic autoimmune disease.

\section{DISCUSSION}

Our study demonstrated that the introduction of the anti-DFS70 antibody test in the initial investigation of patients with suspected systemic autoimmune disease would avoid unnecessary followup visits and minimize the use of health resources generated by suspicion of a potential systemic autoimmune disease.

Anti-DFS70 antibody displays its highest predictive value when its finding is isolated ${ }^{8,12,25}$. Mariz, et $a l^{8}$ reported that none of 40 healthy individuals with isolated anti-DFS70 reactivity developed a systemic autoimmune disease within an average 4-year followup. In our study, a 3-year followup appeared to be sufficient to rule out progression to an overt systemic autoimmune disease, according to previous studies on UCTD natural history $26,27,28,29,30,31$. Fitch-Rogalsky, et $a l^{25}$ reported that anti-DFS70 antibody-positive subjects have a positive likelihood ratio (LR+) of nonsystemic autoimmune rheumatic disease of 5.4 and when considered the $\mathrm{LR}+$ increases to 10.9 when found isolated. These data were recently confirmed by Shovman, et al ${ }^{12}$, demonstrating that prevalence of monospecific anti-DFS70 antibodies was significantly higher in healthy subjects than in patients with ANA-related autoimmune diseases $(10.9 \%$ vs $1.9 \%$, $\mathrm{p}=0.02$ ). Our results are consistent with the literature because none of the control subjects were found to be anti-DFS70 monospecific.

Only homogeneous, speckled, or homogeneous-speckled ANA patterns (AC-2 International Consensus on Autoantibody Patterns patterns) are considered to be consistent with anti-DFS70 antibody. These data easily lead to the conclusion that narrowing the population to which the test is applied increases its potential value. In fact, the most appropriate role of anti-DFS70 antibody screening would be ruling out autoimmune disorders in subjects with low pre-test probability of a systemic autoimmune disease, ANA positive with consistent pattern, and negative ENA or anti-thyroperoxidase antibodies. 

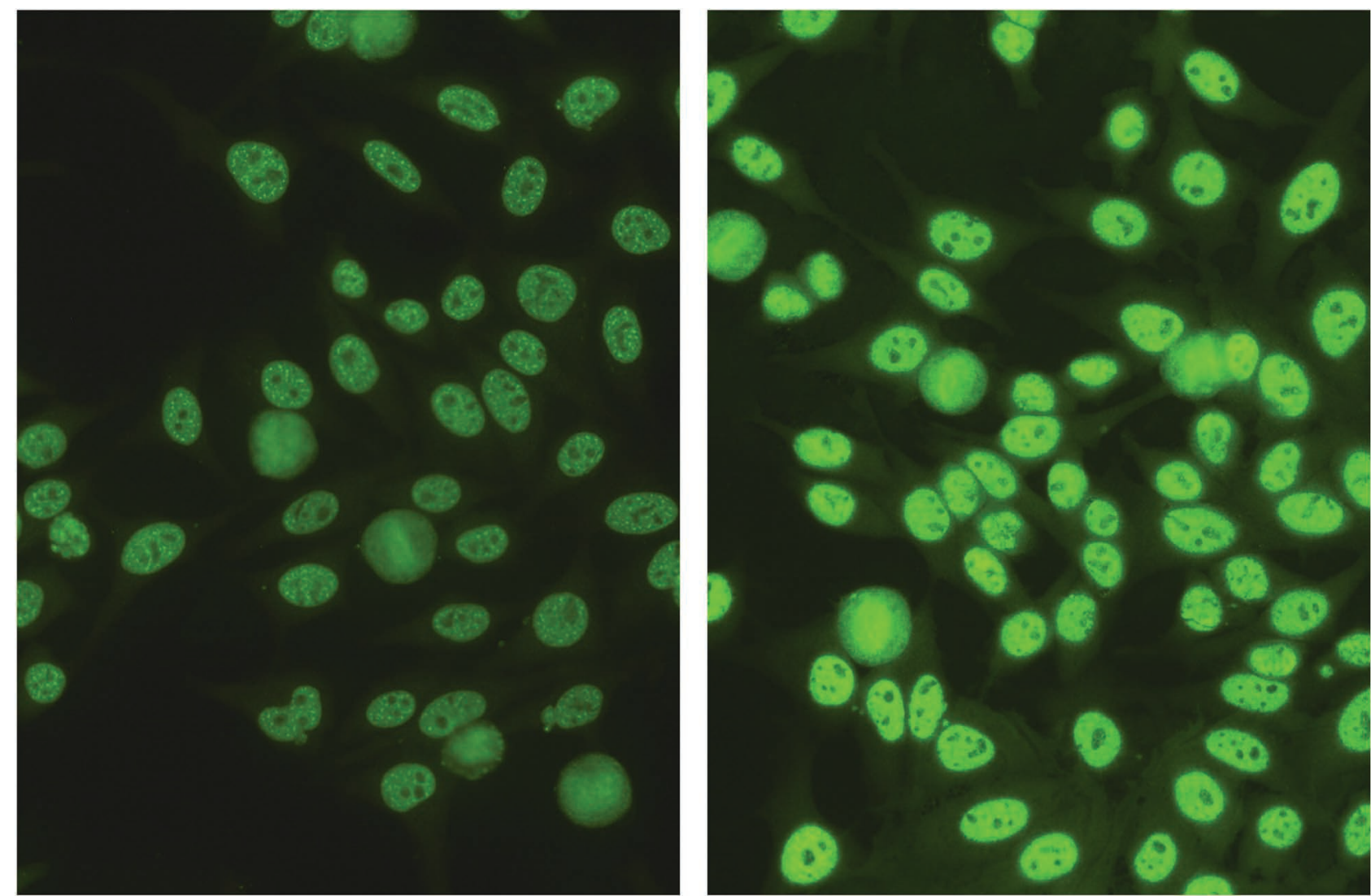

Figure 2. Immunofluorescence pattern on HEp-2 substrate (INOVA Diagnostics). Left: case serum with a DFS70 value of 248.7 and ANA titer 1:80. Right: disease control serum from a patient with Sjögren syndrome, DFS70 value of 237.2, and ANA titer 1:320. DFS: dense fine speckled 70; ANA: antinuclear antibody.

Table 1C. Individual values of Quanta Flash anti-DFS70 assay with respective titer of ANA IIF.

\begin{tabular}{|c|c|c|c|c|c|}
\hline $\begin{array}{l}\text { ANA } \\
\text { Titer }\end{array}$ & Anti-Hep-2 IIF & $\begin{array}{c}\text { Cytoplasmic } \\
\text { Titer }\end{array}$ & $\begin{array}{c}\text { Cytoplasmic } \\
\text { Pattern }\end{array}$ & $\begin{array}{c}\text { Quanta Flash } \\
\text { Anti-DFS70 } \\
\text { Value (CU) }\end{array}$ & Group \\
\hline $1: 80$ & Homogeneous-speckled* & Negative & & 25.8 & Case \\
\hline $1: 320$ & Homogeneous-speckled* & Negative & & 198.8 & Case \\
\hline $1: 80$ & Homogeneous-speckled* & Negative & & 248.7 & Case \\
\hline $1: 160$ & Homogeneous-speckled* & Negative & & 101.5 & Case \\
\hline $1: 320$ & Homogeneous-speckled* & Negative & & 243.6 & Case \\
\hline 1:640 & Homogeneous and speckled & Negative & & 56.4 & SLE \\
\hline 1:640 & Speckled (and homogeneous 1:80) & Negative & & 237.2 & SS \\
\hline 1:640 & Centromeric (and homogeneous $1: 40$ ) & )) $1: 160$ & Granular** & 79.9 & $\mathrm{SSc}$ \\
\hline $1: 160$ & Nucleolar & Negative & & 23.6 & APS \\
\hline
\end{tabular}

\footnotetext{
* The observed pattern is compatible with the description of the DSF described pattern and the suspicion of the presence of anti-DSF70 antibodies. ** This sample is positive by Dot-Blot for AMA-M2. Anti-DFS70: anti-dense fine speckled 70 antibody; ANA: antinuclear antibody; IIF: indirect immunofluorescence; CU: calculated units; SLE: systemic lupus erythematosus; SS: Sjögren syndrome; SSc: systemic sclerosis; APS: antiphospholipid syndrome.
}

Pretest probability of a systemic autoimmune disease strongly depends on clinical judgment, which remains the cornerstone of the diagnosis, but the probability is also influenced by the clinical setting ${ }^{32,33}$. Within populations where the pretest probability of a systemic autoimmune disease is generally low, such as in primary care, the added value of a positive anti-DFS70 antibody test would be worth more to exclude a systemic autoimmune disease, if compared to secondary and tertiary care. Our study was performed in a tertiary-level center, and as expected, overall prevalence of anti-DFS70 antibody was low in all groups. These differences among clinical settings have been recently reproduced

Personal non-commercial use only. The Journal of Rheumatology Copyright @ 2020 . All rights reserved. 
Table 2. Health states probabilities transitions used in the decision tree model and BIA.

Expected Scenario including Anti-DFS70 Screening

Anti-DFS70 + low possibility of developing a systemic autoimmune disease Anti-DFS70, with possibility of developing a systemic autoimmune disease

Evolving to resolution of symptoms after 3 yrs' followup

Remaining with unspecific symptoms after 3 yrs' followup

Developing a systemic autoimmune disease after 3 yrs' followup

SLE

SS

$\mathrm{SSc}$

APS

Current clinical practice scenario (without anti-DSF70 screening)

Anti-DFS70, with possibility of developing a systemic autoimmune disease

Evolving to resolution of symptoms after 3 yrs' followup

Remaining with unspecific symptoms after 3 yrs followup

Developing a systemic autoimmune disease after 3 yrs' followup

SLE
SS
SSc
APS

Probability

Source

$\begin{array}{cc}0.04 & \text { Case-control study } \\ 0.96 & \text { Case-control study } \\ 0.33 & \text { Clinical experts and Bodalay, et } a l^{32} \text {; Danieli, et } a l^{33} \\ 0.33 & \\ 0.33 & \text { Case-control study } \\ 0.30 & \\ 0.27 & \\ 0.22 & \\ 0.22 & \text { Source } \\ \text { Probability } & \text { Current practice } \\ 1.00 & \\ 0.33 & \\ 0.33 & \\ 0.33 & \text { Case-control study } \\ 0.30 & \\ 0.27 & \\ 0.22 & \\ 0.22 & \end{array}$

BIA: budget impact analysis. APS: antiphospholipid syndrome; SLE: systemic lupus erythematosus; SS: Sjögren syndrome; SSc: systemic sclerosis; anti-DFS70: anti-dense fine speckled 70 antibody.

Table 3. Mean cost per patient per clinical scenarios (current and expected) for 3 years.

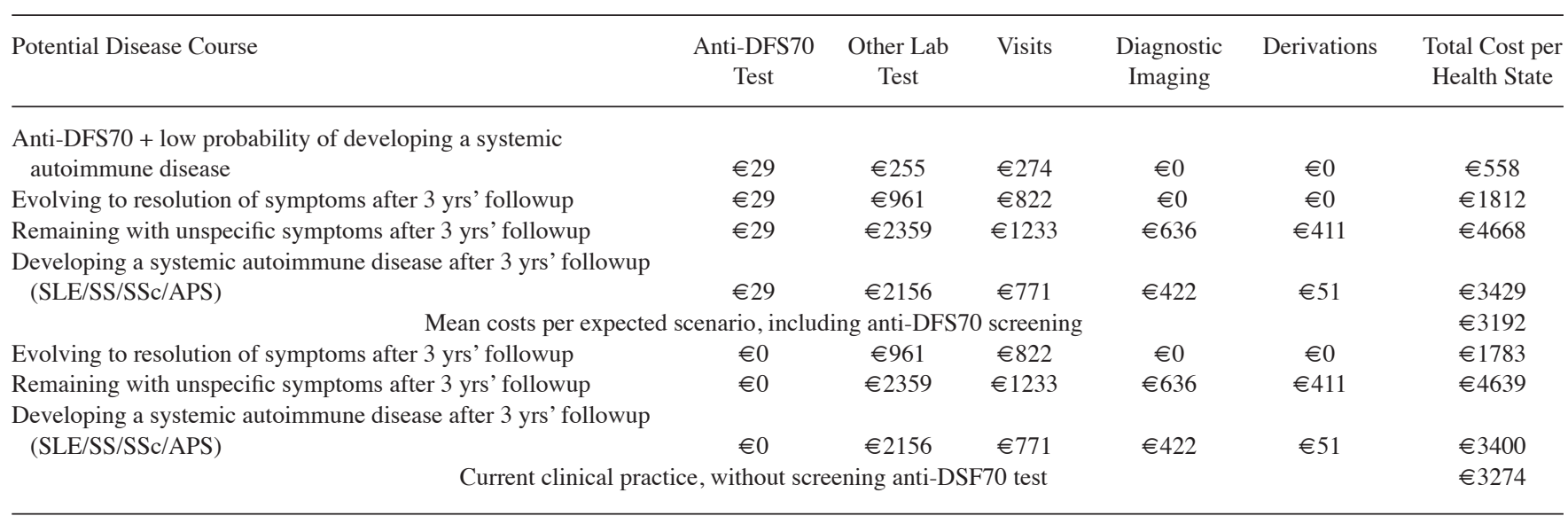

Anti-DFS70: anti-dense fine speckled-70 antibody; APS: antiphospholipid syndrome; SLE: systemic lupus erythematosus; SS: Sjögren syndrome; SSc: systemic sclerosis.

in a Belgian study that compared anti-DFS70 antibody prevalence among sera obtained from primary, secondary, and tertiary care laboratories ${ }^{34}$.

Regarding patients with a defined systemic autoimmune disease, the proportion of anti-DFS70 antibody positivity is similar to those found in SLE $(0-6 \%)^{9,13,35,36,37}$ and SSc $(0.6-2.5 \%)^{9,13,36}$ but significantly lower for SS $(1.2 \%$ vs $11.3 \%)^{9}$. Our choice to introduce APS as a study group can be puzzling, since APS is not generally ANA-related. However, previous reports of potential association between anti-DFS70 antibody and APS are of interest ${ }^{38}$. In our cohort, no difference in either obstetric morbidity or thrombosis incidence has been outlined in the case group. Considering our data together with the qualified literature, the association of anti-DFS70 antibody with APS manifestations appears unfounded.

Regarding the economic perspective, Gundín, et $a l^{11}$ compared a conventional algorithm used at a hospital setting in Spain for the diagnosis of patients with suspicion of ANA-associated autoimmune diseases versus a new algorithm including anti-DFS70 antibody detection. They considered 2 types of costs: the laboratory ANA and followup testing, and the resulting clinic visits. The authors found that the use of the new algorithm resulted in a cost savings of $€ 60,869$. The incidence of positive anti-DFS70 antibodies was reported in $12.7 \%$ for the total population under study. This finding would be in line with our sensitivity analysis, in which we demonstrated that at a higher incidence of positive 
Table 4. Budget impact analysis.

Current Scenario: Clinical Practice

\begin{tabular}{|c|c|c|c|}
\hline & $\begin{array}{c}\text { Year } 1 \\
\mathrm{n}=124\end{array}$ & Year 2 & Year 3 \\
\hline Patients with low probability of developing systemic autoimmune disease & $€ 0$ & $€ 0$ & $€ 0$ \\
\hline Cost of other laboratory test & $€ 0$ & $€ 0$ & $€ 0$ \\
\hline Cost of diagnostic imaging & $€ 0$ & $€ 0$ & $€ 0$ \\
\hline \multirow[t]{2}{*}{ Cost of derivations } & $€ 0$ & $€ 0$ & $€ 0$ \\
\hline & $\begin{array}{c}\text { Year } 1 \\
\mathrm{n}=124\end{array}$ & Year 2 & Year 3 \\
\hline Cost of other laboratory test & $€ 75,441$ & $€ 75,441$ & $€ 75,441$ \\
\hline Cost of visits & $€ 38,931$ & $€ 38,931$ & $€ 38,931$ \\
\hline Cost of diagnostic imaging & $€ 14,580$ & $€ 14,580$ & $€ 14,580$ \\
\hline Cost of derivations & $€ 6371$ & $€ 6371$ & $€ 6371$ \\
\hline Total costs, current scenario: clinical practice & $€ 135,323$ & $€ 135,323$ & $€ 135,323$ \\
\hline
\end{tabular}

Expected Scenario, including Anti-DFS70 Screening Test

Patients with low probability of developing systemic autoimmune disease

Year $1, \mathrm{n}=5$
$€ 2791$
$€ 145$
$€ 1276$
$€ 1370$
$€ 0$
$€ 0$
Year 1
$€ 133,317$
$€ 3451$
$€ 72,399$
$€ 37,361$
$€ 13,992$
$€ 6114$
$€ 136,108$
$€ 785$

$\begin{array}{cc}\text { Year } 2, \mathrm{n}=0 & \text { Year } 3, \mathrm{n}=0 \\ € 0 & € 0 \\ € 0 & € 0 \\ € 0 & € 0 \\ € 0 & € 0 \\ € 0 & € 0 \\ € 0 & € 0 \\ \text { Year } 2 & \text { Year } 3 \\ \mathrm{n}=119 & \\ € 129,866 & € 129,866 \\ € 0 & € 0 \\ € 72,399 & € 72,399 \\ € 37,361 & € 37,361 \\ € 13,992 & € 13,992 \\ € 6114 & € 6114 \\ € 129,866 & € 129,866 \\ -€ 5457 & -€ 5457\end{array}$

Patients with probability of developing systemic autoimmune disease

Cost of anti-DFS70 diagnostic test

Cost of anti-DFS70 diagnostic test

Cost of other laboratory test

Cost of visits

Cost of diagnostic imaging

Cost of derivations

Cost of other laboratory test

Cost of visits

Cost of diagnostic imaging

Cost of derivations

Total costs, expected sequence including anti-DFS70 screening test Budget impact $€ 785$

Year $2, \mathrm{n}=0$
$€ 0$
$€ 0$
$€ 0$
$€ 0$
$€ 0$
$€ 0$
Year 2
$\mathrm{n}=119$
$€ 129,866$
$€ 0$
$€ 72,399$
$€ 37,361$
$€ 13,992$
$€ 6114$
$€ 129,866$
$-€ 5457$

Values are 2018 Euros. Anti-DFS70: anti-dense fine speckled 70 antibody.

\begin{tabular}{|c|c|}
\hline \multirow[t]{4}{*}{ Low } & cost anti DFS70 test $(23,20 €-34,80 €)$ \\
\hline & $\%$ remaining with unspecific symtom( $(40 \%-26,7 \%)$ \\
\hline & $\%$ evolving to resolution $(26,7 \%-40 \%)$ \\
\hline & $\begin{array}{l}\text { \% Developing a systemic autoimmune disease } \\
(26,7 \%-40 \%)\end{array}$ \\
\hline
\end{tabular}

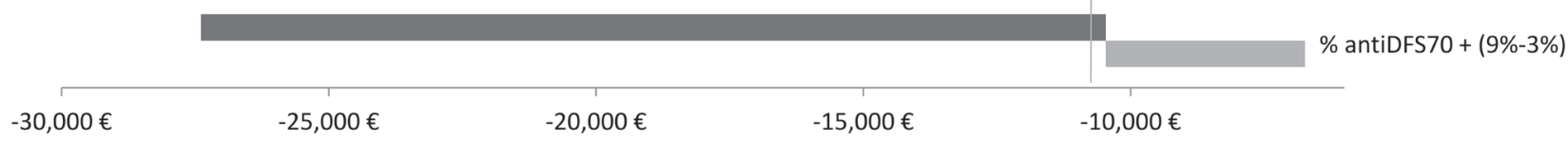

Figure 3. Budget impact sensitivity analysis with a tornado diagram (€10,128 base case). Anti-DFS70: anti-dense fine speckled 70. 
anti-DFS70 antibody tests, the economic effect would be greater. In fact, prevalence of anti-DFS70 positivity is the key variable of the model, suggesting even greater savings if implemented in settings such as primary care, where it is likely to be higher (tornado diagram, Figure 3). This is undoubtedly the real strength of our study.

The main limitation of our study is the observational design that did not allow having a real but only "simulated" timeline. Further, additional groups of patients with other systemic autoimmune diseases (e.g., rheumatoid arthritis, autoimmune inflammatory myopathies, antineutrophil cytoplasmic antibody-associated vasculitis) should be considered as control groups. Future prospective studies would clarify whether our data are confirmed.

Isolated anti-DFS70 antibody test represents a potential biomarker that can be used clinically to discriminate systemic autoimmune diseases from other conditions in ANA-positive individuals - better if used in a proper referral context and would help avoid unnecessary followup visits and costs. For that reason, algorithms containing anti-DFS70 antibody tests will allow for the reduction of unnecessary tests and followup visits generated by the suspicion of an unlikely autoimmune disease in the upcoming years.

\section{DATA SHARING POLICY}

Unidentified and additional raw data that form the basis for this work can be requested through correspondence with the contact author, and under the extent possible according to Spanish law.

\section{REFERENCES}

1. Agmon-Levin N, Damoiseaux J, Kallenberg C, Sack U, Witte T, Herold $\mathrm{M}$, et al. International recommendations for the assessment of autoantibodies to cellular antigens referred to as anti-nuclear antibodies. Ann Rheum Dis 2014;73:17-23.

2. Ochs RL, Mahler M, Basu A, Rios-Colon L, Sanchez TW, Andrade LE, et al. The significance of autoantibodies to DFS70/LEDGFp75 in health and disease: integrating basic science with clinical understanding. Clin Exp Med 2016;16:273-93.

3. Chan EK, Damoiseaux J, de Melo Cruvinel W, Carballo OG, Conrad K, Francescantonio PL, et al. Report on the second International Consensus on ANA Pattern (ICAP) workshop in Dresden 2015. Lupus 2016;25:797-804.

4. Damoiseaux J, von Muhlen CA, Garcia-De La Torre I, Carballo OG, de Melo Cruvinel W, Francescantonio PL, et al. International consensus on ANA patterns (ICAP): the bumpy road towards a consensus on reporting ANA results. Auto Immun Highlights 2016;7:1.

5. Miyara M, Albesa R, Charuel JL, El Amri M, Fritzler MJ, Ghillani-Dalbin P, et al. Clinical phenotypes of patients with anti-DFS70/LEDGF antibodies in a routine ANA referral cohort. Clin Dev Immunol 2013;2013:703759.

6. Bizzaro N, Tonutti E, Tampoia M, Infantino M, Cucchiaro F, Pesente F, et al; Study Group on Autoimmune Diseases of the Italian Society of Laboratory Medicine. Specific chemoluminescence and immunoasdorption tests for anti-DFS70 antibodies avoid false positive results by indirect immunofluorescence. Clin Chim Acta 2015;451:271-7.

7. Mahler M, Parker T, Peebles CL, Andrade LE, Swart A, Carbone
Y, et al. Anti-DFS70/LEDGF antibodies are more prevalent in healthy individuals compared to patients with systemic autoimmune rheumatic diseases. J Rheumatol 2012;39:2104-10.

8. Mariz HA, Sato EI, Barbosa SH, Rodrigues SH, Dellavance A, Andrade LE. Pattern on the antinuclear antibody-HEp-2 test is a critical parameter for discriminating antinuclear antibody-positive healthy individuals and patients with autoimmune rheumatic diseases. Arthritis Rheum 2011;63:191-200.

9. Muro Y, Sugiura K, Morita Y, Tomita Y. High concomitance of disease marker autoantibodies in anti-DFS70/LEDGF autoantibody-positive patients with autoimmune rheumatic disease. Lupus 2008;17:171-6.

10. Bentow C, Fritzler MJ, Mummert E, Mahler M. Recognition of the dense fine speckled (DFS) pattern remains challenging: results from an international internet-based survey. Auto Immun Highlights 2016;7:8.

11. Gundín S, Irure-Ventura J, Asensio E, Ramos D, Mahler M, Martínez-Taboada V, et al. Measurement of anti-DFS70 antibodies in patients with ANA-associated autoimmune rheumatic diseases suspicion is cost-effective. Auto Immun Highlights 2016;7:10.

12. Shovman O, Gilburd B, Chayat C, Amital H, Langevitz P, Watad A, et al. Prevalence of anti-DFS70 antibodies in patients with and without systemic autoimmune rheumatic diseases. Clin Exp Rheumatol 2018;36:121-6.

13. Watanabe A, Kodera M, Sugiura K, Usuda T, Tan EM, Takasaki Y, et al. Anti-DFS70 antibodies in 597 healthy hospital workers. Arthritis Rheum 2004;50:892-900.

14. Clegg DO, Williams HJ, Singer JZ, Steen VD, Schlegel S, Ziminski $\mathrm{C}$, et al. Early undifferentiated connective tissue disease. II. The frequency of circulating antinuclear antibodies in patients with early rheumatic diseases. J Rheumatol 1991;18:1340-3.

15. Mosca M, Neri R, Bombardieri S. Undifferentiated connective tissue diseases (UCTD): a review of the literature and a proposal for preliminary classification criteria. Clin Exp Rheumatol 1999;17:615-20.

16. Mosca M, Tani C, Carli L, Bombardieri S. Undifferentiated CTD a wide spectrum of autoimmune diseases. Best Pract Res Clin Rheumatol 2012;26:73-7.

17. Doria A, Mosca M, Gambari PF, Bombardieri S. Defining unclassifiable connective tissue diseases: incomplete, undifferentiated, or both? J Rheumatol 2005;32:213-5.

18. Petri M, Orbai AM, Alarcon GS, Gordon C, Merrill JT, Fortin PR, et al. Derivation and validation of the Systemic Lupus International Collaborating Clinics classification criteria for systemic lupus erythematosus. Arthritis Rheum 2012;64:2677-86.

19. Shiboski CH, Shiboski SC, Seror R, Criswell LA, Labetoulle M, Lietman TM, et al; International Sjögren's Syndrome Criteria Working Group. 2016 American College of Rheumatology/ European League Against rheumatism classification criteria for primary Sjogren's syndrome: A consensus and data-driven methodology involving three international patient cohorts. Arthritis Rheumatol 2017;69:35-45.

20. van den Hoogen F, Khanna D, Fransen J, Johnson SR, Baron M, Tyndall A, et al. 2013 classification criteria for systemic sclerosis: an American College of Rheumatology/European League against Rheumatism collaborative initiative. Arthritis Rheum 2013;65:2737-47.

21. Miyakis S, Lockshin MD, Atsumi T, Branch DW, Brey RL, Cervera $\mathrm{R}$, et al. International consensus statement on an update of the classification criteria for definite antiphospholipid syndrome (APS). J Thromb Haemost 2006;4:295-306.

22. York Health Economics Consortium (YHEC). Decision tree. [Internet. Accessed March 10, 2020.] Available from: www.yhec. co.uk/glossary/decision-tree

23. Mosca M, Tani C, Talarico R, Bombardieri S. Undifferentiated

Personal non-commercial use only. The Journal of Rheumatology Copyright $\subset$ 2020. All rights reserved. 
connective tissue diseases (UCTD): Simplified systemic autoimmune diseases. Autoimmun Rev 2011;10:256-8.

24. York Health Economics Consortium (YHEC). Sensitivity analysis. [Internet. Accessed March 10, 2020.] Available from: www.yhec. co.uk/glossary/sensitivity-analysis

25. Fitch-Rogalsky C, Steber W, Mahler M, Lupton T, Martin L, Barr $\mathrm{SG}$, et al. Clinical and serological features of patients referred through a rheumatology triage system because of positive antinuclear antibodies. PLoS One 2014;9:e93812.

26. Lom-Orta H, Alarcon-Segovia D, Diaz-Jouanen E. Systemic lupus erythematosus. Differences between patients who do, and who do not, fulfill classification criteria at the time of diagnosis. J Rheumatol 1980;7:831-7.

27. Greer JM, Panush RS. Incomplete lupus erythematosus. Arch Intern Med 1989;149:2473-6.

28. Mosca M, Neri R, Bencivelli W, Tavoni A, Bombardieri S. Undifferentiated connective tissue disease: analysis of 83 patients with a minimum followup of 5 years. J Rheumatol 2002;29:2345-9.

29. Swaak AJ, van de Brink H, Smeenk RJ, Manger K, Kalden JR, Tosi S, et al; Study group on incomplete SLE and SLE with disease duration longer than 10 years. Incomplete lupus erythematosus: results of a multicentre study under the supervision of the EULAR Standing Committee on International Clinical Studies Including Therapeutic Trials (ESCISIT). Rheumatol 2001;40:89-94.

30. Bodolay E, Csiki Z, Szekanecz Z, Ben T, Kiss E, Zeher M, et al. Five-year follow-up of 665 Hungarian patients with undifferentiated connective tissue disease (UCTD). Clin Exp Rheumatol 2003;21:313-20.
31. Danieli MG, Fraticelli P, Franceschini F, Cattaneo R, Farsi A, Passaleva A, et al. Five-year follow-up of 165 Italian patients with undifferentiated connective tissue diseases. Clin Exp Rheumatol 1999; 17:585-91.

32. Abeles AM, Abeles M. The clinical utility of a positive antinuclear antibody test result. Am J Med 2013;126:342-8.

33. Avery TY, van de Cruys M, Austen J, Stals F, Damoiseaux JG. Anti-nuclear antibodies in daily clinical practice: prevalence in primary, secondary, and tertiary care. J Immunol Res 2014;2014:401739.

34. Bonroy C, Schouwers S, Berth M, Van Hoovels L. The importance of detecting anti-DFS70 in routine clinical practice: comparison of different care settings. Clin Chem Lab Med 2019;57:e47-8.

35. Choi MY, Clarke AE, St Pierre Y, Hanly JG, Urowitz MB, Romero-Diaz J, et al. The prevalence and determinants of anti-DFS70 autoantibodies in an international inception cohort of systemic lupus erythematosus patients. Lupus 2017;26:1051-9.

36. Ochs RL, Muro Y, Si Y, Ge H, Chan EK, Tan EM. Autoantibodies to DFS $70 \mathrm{kd} /$ transcription coactivator p75 in atopic dermatitis and other conditions. J Allergy Clin Immunol 2000;105:1211-20.

37. Mahler M, Hanly JG, Fritzler MJ. Importance of the dense fine speckled pattern on HEp-2 cells and anti-DFS70 antibodies for the diagnosis of systemic autoimmune diseases. Autoimmun Rev 2012;11:642-5.

38. Marlet J, Ankri A, Charuel JL, Ghillani-Dalbin P, Perret A, Martin-Toutain I, et al. Thrombophilia associated with anti-DFS70 autoantibodies. PLoS One 2015;10:e0138671. 